\title{
Um Método Simples para Codificação de Imagens por Mapa de Significância
}

\author{
Marcos Ricardo A. Morais, Francisco Madeiro e Elmar Uwe K. Melcher
}

\begin{abstract}
Resumo-O presente trabalho apresenta um método simples para codificação de imagens baseada em mapa de significância. Apesar da simplicidade, o método apresenta uma superioridade sobre JPEG. Os resultados obtidos mostram que a codificação eficiente do mapa é essencial em compressão de imagens com transformadas de bloco.
\end{abstract}

Palavras-Chave-Compressão de sinais, codificação de imagem, mapa de significância.

Abstract-This work presents a simple method for image coding based on significance map. Although simple, the method overperforms JPEG. Results show that efficient coding of the map is essential in image compression based on block transform. map.

Keywords-Signal compression, image coding, significance

\section{INTRODUÇÃO}

Apesar do aumento observado na capacidade de armazenamento e de transmissão dos equipamentos mais modernos, observa-se a exigência de métodos cada vez mais eficientes de compressão de sinais de multimídia. Nesse contexto estão as aplicações de comunicações móveis audiovisuais, a televisão e o DVD de alta definição, o cinema digital, etc. A busca de métodos mais eficientes de codificação tem promovido um grande avanço nos domínios do processamento digital de sinais e da teoria da informação. Avanços significativos estão associados ao uso de transformadas para a codificação de imagem, áudio e vídeo. Para o caso de vídeo, o padrão H.264/AVC/MPEG-4 parte 10 inovou nos métodos de estimação e compensação de movimento, transformada de bloco reversível e de tamanho reduzido, divisão e codificação hierárquica dos macroblocos, com o uso de um codificador aritmético como estágio final de codificação.

Um bloco de coeficientes, obtidos por meio da aplicação de transformadas de bloco em uma imagem, pode ser representado por um mapa de significância, que indica que elementos são não nulos, e por suas respectivas magnitudes e sinais.

Este artigo apresenta um método simples para codificação de imagens baseado em mapa de significância.

Marcos Ricardo A. Morais, Universidade Federal de Campina Grande, Campina Grande, PB, Brasil, e-mail: morais@ dee.ufcg.edu.br. Francisco Madeiro, Escola Politécnica de Pernambuco - Universidade de Pernambuco, Recife, PE, Brasil, e-mail: franciscomadeiro@yahoo.com.br. Elmar Uwe K Melcher, Universidade Federal de Campina Grande, Campina Grande, PB, Brasil, e-mail: elmar@dsc.ufcg.edu.br. Francisco Madeiro e Elmar Melcher também estão com o Instituto de Estudos Avançados em Comunicações (Iecom), Campina Grande, PB, Brasil.
Na seção a seguir, investigamos a codificação baseada na separação entre coeficientes nulos e não nulos através de um mapa de significância. Por meio de um algoritmo muito simples de codificação baseado em mapa de significância, observamos a proporção de bits gastos para codificar a significância, o sinal e a amplitude dos coeficientes.

\section{CODIFICAÇÃO POR MAPA DE SIGNIFICÂNCIA}

Na codificação de imagem por transformada, os coeficientes quantizados formam um conjunto de dados que representam a imagem. A este conjunto de dados aplica-se um codificador entrópico. $\mathrm{O}$ conjunto de dados possui uma estrutura particular que pode ser aproveitada de forma a melhorar a codificação entrópica. Especificamente, no caso das transformadas de bloco, como a DCT, aplicadas a imagens naturais, os coeficientes tendem a possuir maior amplitude quando sua freqüência espacial, horizontal ou vertical, é menor. Essa estrutura pode ser vista como uma redundância intra-bloco.

Outra estrutura que pode ser considerada no projeto de um codificador é o fato de a transformada de bloco dividir o plano tempo-freqüência (no caso de imagem, espaço-freqüência), em regiões, denominadas sub-bandas, de mesma área. A posição do coeficiente no bloco transformado indica sua freqüência espacial. Os coeficientes de mesma posição de blocos adjacentes possuem, devido à regularidade e à localidade encontradas nas imagens naturais, valores correlacionados. Um codificador pode aproveitar essa redundância inter-blocos.

Podemos considerar a concepção de métodos que procurem explorar de forma conjunta as redundâncias intra e interblocos. O conceito envolveria encontrar alguma correlação entre blocos baseada em alguma característica de cada bloco. Um exemplo deste princípio poderia ser a busca de blocos que possuam mapas de significância de coeficientes transformados semelhantes.

O fato de estarmos explorando uma estrutura de mais alto nível, que abrange as dependências inter e intra-blocos, nos dá a esperança de conseguirmos algum ganho de codificação, com relação às técnicas tradicionais. No caso de vídeo, podemos ainda esperar que a exploração conjunta da redundância temporal inter-quadros promova, também, ganho de codificação. Cabe salientar que a codificação da imagem resíduo realizada desta forma, utilizando quadros anteriores como referência, não segue completamente o paradigma usual na codificação híbrida de vídeo. No modelo usual, procura-se reduzir a redundância temporal através da estimação e compensação de 
movimento, e o resíduo é codificado sem nenhuma referência aos demais quadros.

Analisamos a seguir a separação entre coeficientes nulos e não-nulos por meio do uso de mapa de significância.

\section{A. Mapa de significância}

Coeficientes nulos são gerados pela etapa de quantização, quando os coeficientes da transformada, com módulos menores que um determinado valor, são mapeados em zero. Assim, muitos algoritmos tratam o símbolo zero de forma especial e, implícita ou explicitamente, codificam um mapa de significância. Lembramos que um elemento é dito significante se for não nulo.

Um mapa binário de significância pode ser construído explicitamente. A Fig. 1 mostra o mapa de significância para a DCT $8 \times 8$ da imagem Lena (apresentada na Fig. 2), em que os coeficientes foram quantizados uniformemente (divididos e arredondados) pelo fator de qualidade $Q=150$. Observase claramente o agrupamento de coeficientes significantes ao redor da posição DC. Um fato importante a se observar é a correlação existente entre os blocos próximos. Isto ocorre devido à regularidade e localidade das imagens, que levam à semelhança na resposta espectral de blocos vizinhos.

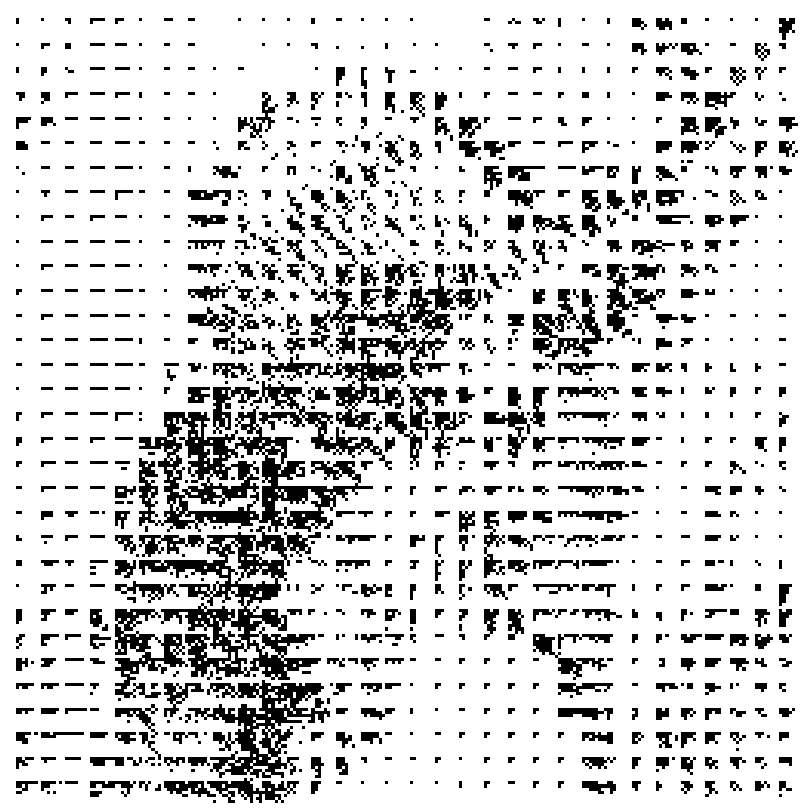

Fig. 1. Mapa de significância para a imagem Lena $256 \times 256$ [1].

Um bloco de coeficientes quantizados pode ser descrito por meio do seu mapa de significância e da amplitude e sinal dos elementos não nulos. Esses elementos podem ser codificados separadamente ou de forma conjunta. Observamos que nos codificadores baseados em comprimento de seqüência de zeros, como o JPEG, a amplitude é codificada conjuntamente com o número de zeros, haja visto que esses valores são correlacionados. Nos codificadores baseados em contexto, como o ARL (Adaptive Run Length) [2], estes fatores são

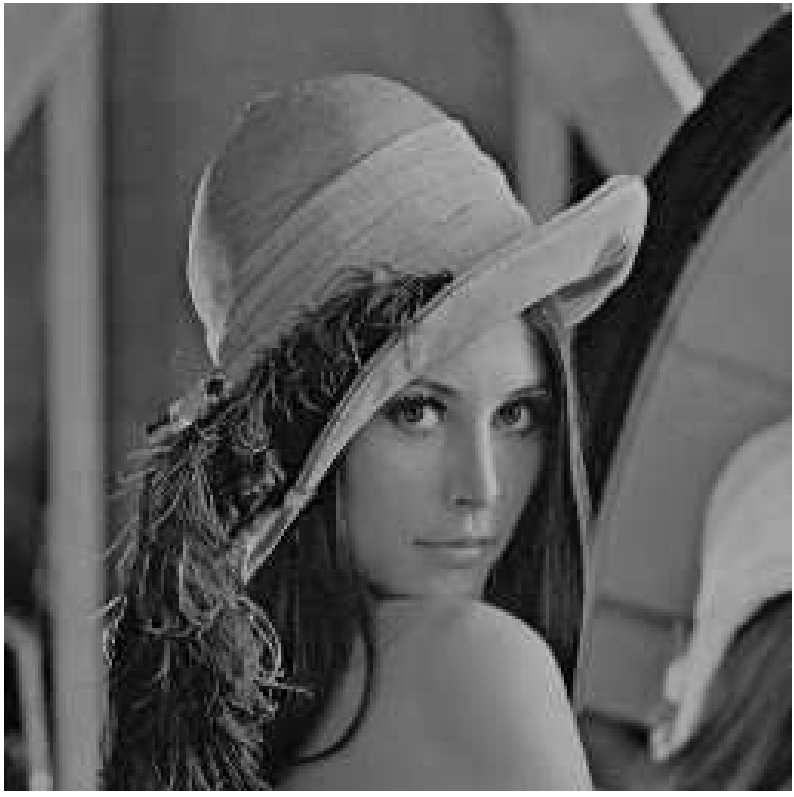

Fig. 2. Imagem Lena $256 \times 256$.

codificados separadamente, permitindo uma melhor adaptação dos contextos.

A seguir introduzimos um algoritmo de codificação simples, baseado na codificação aritmética de contextos [3], em que os fatores representativos dos coeficientes, quais sejam significância, sinal e amplitude, são codificados separadamente e o número de bits relativo a cada fator é medido.

\section{AlgoritMo DE UM CODIFICADOR SIMPLES POR MAPA DE SIGNIFICÂNCIA}

Para observarmos com clareza o número de bits utilizados na codificação de cada um dos elementos que formam um bloco de coeficientes, introduzimos o algoritmo baseado na codificação aritmética de contextos, que pode ser visto na Fig. 3. Cada uma das codificações realizadas nos passos 3, 5 e 6 do algoritmo utiliza um contexto diferente. Utilizamos um codificador aritmético binário adaptativo realizado de acordo com a descrição no Apêndice. A significância e o sinal, que são elementos binários, são codificados diretamente. O valor absoluto dos coeficientes é binarizado como descrito no Apêndice. Antes da codificação, subtraímos 1 do valor do coeficiente quantizado para reduzir sua faixa dinâmica. Isto é possível porque sabemos que o coeficiente é significativo, logo seu valor é pelo menos 1 . O valor original é restaurado no decodificador.

\section{Resultados}

As Tabelas I e II apresentam resultados da aplicação do algoritmo às imagens Barbara (mostrada na Fig. 4) e Lena, para algumas taxas, utilizando DCT. Para cada taxa está discriminado o número de bits usado na codificação de cada fator constituinte. As tabelas também apresentam a qualidade 


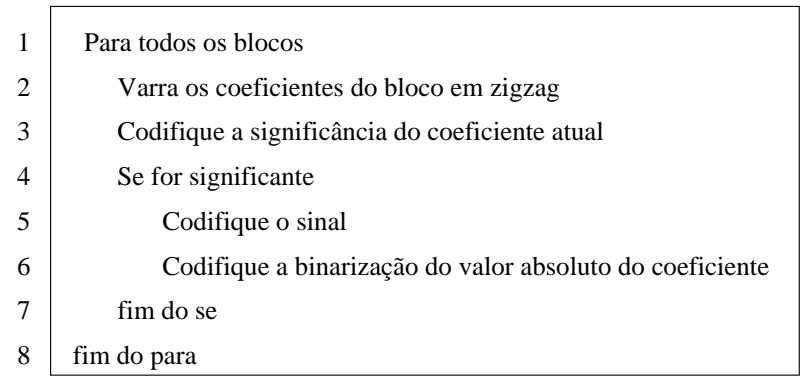

Fig. 3. Algoritmo para análise do uso de bits na codificação de significância, sinal e amplitude.

objetiva em termos de razão sinal-ruído de pico (PSNR, Peak Signal to Noise Ratio). É interessante observar que mesmo esse algoritmo muito simples produz resultados melhores do que o JPEG, para todas as taxas medidas para a imagem Barbara: ganhos de 1,65 dB, 1,39 dB e 1,11 dB são obtidos ao se substituir JPEG pelo método considerado no presente trabalho, para as taxas de codificação de $1,00 \mathrm{bpp}, 0,5 \mathrm{bpp}$ e $0,25 \mathrm{bpp}$ respectivamente. Para a imagem Lena, um ganho de 0,52 dB é obtido ao se substituir JPEG pelo método considerado no presente trabalho, para a taxa de codificação de 1,00 bpp. A superioridade sobre JPEG se deve à capacidade de adaptação da codificação aritmética por contextos.

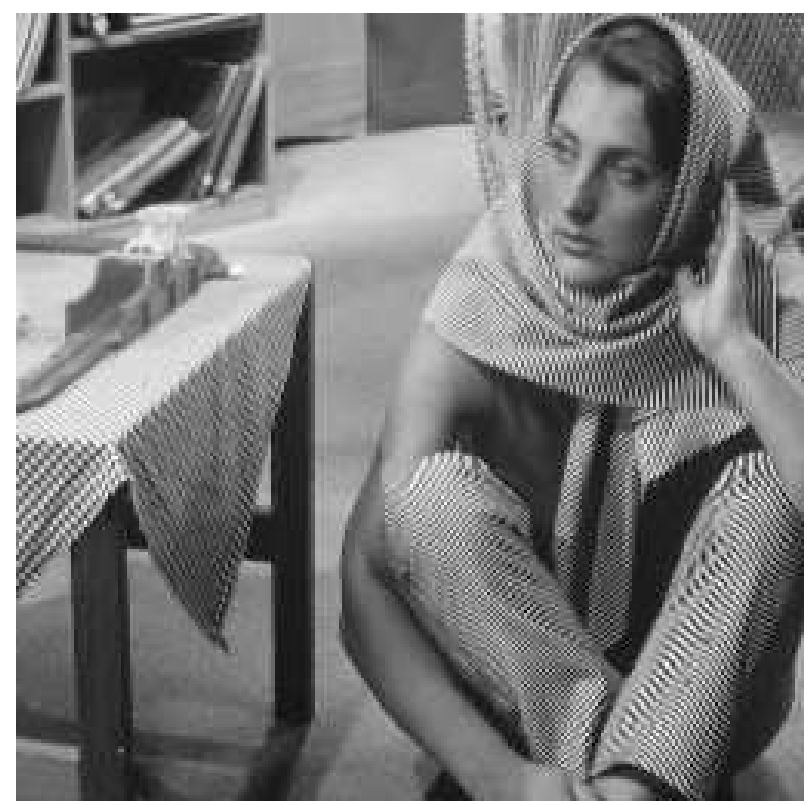

Fig. 4. Imagem Barbara $256 \times 256$.

A codificação separada dos fatores revela que a maior parte dos bits é gasta na representação do mapa de significância, para várias taxas. $\mathrm{O}$ sinal dos coeficientes transformados não apresenta estrutura simples e é normalmente considerado como incompressível, com entropia perto de 1 bit/símbolo. Esta suposição é especialmente válida quando a transformada é ortogonal, como a DCT. A codificação do sinal dos coeficientes transformados foi estudada em detalhes por Deever e
Hernani [4]. No trabalho, através de um processo complexo de projeção de coeficientes e para transformadas biortogonais, foi possível comprimir marginalmente a representação do sinal. Da mesma forma, considera-se que os valores absolutos dos coeficientes significativos não estão correlacionados. Esses valores absolutos são de difícil compressão e não há ganho significativo com a aplicação de contextos ou modelos mais elaborados.

Os resultados das Tabelas I e II mostram que a codificação eficiente do mapa de significância é essencial na compressão de imagens com transformada de bloco.

\section{CONCLUSÃO}

$\mathrm{O}$ presente trabalho apresentou um método simples para codificação de imagens por mapa de significância. $\mathrm{O}$ método apresentado é baseado em codificação aritmética de contextos e, apesar da simplicidade, apresenta uma superioridade sobre JPEG. Para a imagem Barbara, por exemplo, para a taxa de codificação de 0,25 bpp, um ganho de $1,11 \mathrm{~dB}$, em termos de razão sinal-ruído de pico da imagem reconstruída, é obtido ao se substituir JPEG pelo método apresentado neste trabalho.

\section{APÊNDICE}

É possível simplificarmos o codificador aritmético se, além de utilizarmos uma aritmética de precisão reduzida, utilizarmos um número menor de símbolos [5]. No caso extremo, podemos utilizar apenas dois símbolos, o que é denotado como codificador aritmético binário. Nesse caso, o particionamento do intervalo atual quando um novo símbolo é emitido corresponde apenas a uma multiplicação pelo valor da probabilidade deste símbolo. A definição e a modelagem dos contextos também é simplificada, porque é necessário produzir apenas um valor de probabilidade (a outra probabilidade é calculada de forma que a soma seja 1), para cada contexto. A adaptação dos contextos também é muito simples, porque não é necessário armazenar e analisar a história dos símbolos emitidos através de uma estrutura de dados complexa. Essas simplificações levaram ao desenvolvimento de alguns codificadores aritméticos muito eficientes, em termos de velocidade de compressão e descompressão e utilização de memória, com uma perda de eficiência muito pequena na compressão [6]-[8].

Para utilizarmos um codificador aritmético binário com uma fonte com mais de 2 símbolos, é necessário realizarmos um passo de binarização. A binarização corresponde a representar os símbolos da fonte com vários símbolos binários de forma que o símbolo possa ser recuperado no decodificador. A forma mais simples de binarização é representar os $M$ símbolos da fonte com um código binário de comprimento fixo de dimensão $N=\left\lceil\log _{2} M\right\rceil$. Porém, com esta binarização não há uma forma simples de definição dos contextos, haja visto que na representação binária convencional os bits individuais não são correlacionados.

Para a codificação de fontes em que os símbolos são números, e os números de menor valor são mais prováveis, 
TABELA I

ANÁLISE DA PROPORÇÃO DE BITS UTILIZADOS PARA REPRESENTAR SIGNIFICÂNCIA, SINAL E AMPLITUDE, NA CODIFICAÇÃO DA IMAGEM BARBARA, UTILIZANDO DCT.

\begin{tabular}{|c||c|c|c|c|c|c|c|c|}
\hline $\begin{array}{c}\text { Taxa } \\
\text { (bpp) }\end{array}$ & $\begin{array}{c}\text { Razão } \\
\text { Comp. }\end{array}$ & $\begin{array}{c}\text { Coefic. } \\
\text { signif. }\end{array}$ & $\begin{array}{c}\text { Codific. } \\
\text { Amplitude }\end{array}$ & $\begin{array}{c}\text { Codific. } \\
\text { Sinal }\end{array}$ & $\begin{array}{c}\text { Codific. } \\
\text { Signif. }\end{array}$ & $\begin{array}{c}\text { Signif./total } \\
(\%)\end{array}$ & PSNR (dB) & $\begin{array}{c}\text { PSNR (dB) } \\
\text { JPEG }\end{array}$ \\
\hline \hline 1,00 & $1: 8$ & 46.052 & 10.424 & 5.987 & 16.460 & 50,0 & 34,90 & 33,25 \\
\hline 0,50 & $1: 16$ & 22.455 & 3.541 & 2.922 & 9.916 & 60,6 & 29,88 & 28,49 \\
\hline 0,25 & $1: 32$ & 10.875 & 1.288 & 1.424 & 5.484 & 66,9 & 26,21 & 25,10 \\
\hline
\end{tabular}

TABELA II

ANÁLISE DA PROPORÇÃo DE BITS UTILIZADOS PARA REPRESENTAR SIGNIFICÂNCIA, SINAL E AMPLITUDE, NA CODIFICAÇÃO DA IMAGEM LENA, UTILIZANDO DCT.

\begin{tabular}{|c||c|c|c|c|c|c|c|c|}
\hline $\begin{array}{c}\text { Taxa } \\
\text { (bpp) }\end{array}$ & $\begin{array}{c}\text { Razão } \\
\text { Comp. }\end{array}$ & $\begin{array}{c}\text { Coefic. } \\
\text { signif. }\end{array}$ & $\begin{array}{c}\text { Codific. } \\
\text { Amplitude }\end{array}$ & $\begin{array}{c}\text { Codific. } \\
\text { Sinal }\end{array}$ & $\begin{array}{c}\text { Codific. } \\
\text { Signif. }\end{array}$ & $\begin{array}{c}\text { Signif./total } \\
(\%)\end{array}$ & PSNR (dB) & $\begin{array}{c}\text { PSNR (dB) } \\
\text { JPEG }\end{array}$ \\
\hline \hline 1,00 & $1: 8$ & 44.574 & 10.462 & 5.788 & 16.626 & 50,6 & 38,48 & 37,96 \\
\hline 0,50 & $1: 16$ & 21.505 & 4.833 & 2.792 & 8.775 & 53,6 & 34,95 & 34,86 \\
\hline 0,25 & $1: 32$ & 10.828 & 2.090 & 1.401 & 4.718 & 57,6 & 31,40 & 31,40 \\
\hline
\end{tabular}

uma binarização que tem se mostrado muito eficiente é representar o símbolo como uma seqüência de símbolos 0 de comprimento igual ao seu valor numérico seguido por um símbolo 1 [9]-[11]. Esse esquema é conhecido como código unário. O código unário é um dos mais simples códigos utilizados na codificação de sequiências de números inteiros.

A codificação de sequiências de números inteiros com determinadas distribuições é amplamente estudada. Para algumas distribuições já foram encontrados, de forma fechada, códigos ótimos, ou seja, códigos com comprimento médio igual à entropia. A utilização desses códigos permite chegar aos mesmos resultados em termos de comprimento médio de código do que a utilização da codificação aritmética. Esses códigos são normalmente muito simples de serem construídos e nesses casos podem substituir o código aritmético. Estes códigos também podem ser utilizados como esquemas de binarização para aplicação de um código aritmético binário. Nesse caso, em um esquema híbrido, é possível, através do uso de contextos, uma melhor adaptação à fonte.

A perda de desempenho do código aritmético binário com relação a um código multi-símbolos é marginal [12].

\section{REFERÊNCIAS}

[1] M. R. A. Morais. "Codificação de Imagem e Vídeo com Casamento Parcial de Padrões em Transformadas de Bloco". Ph.D. thesis, Universidade Federal de Campina Grande, COPELE, Campina Grande, PB, 2004.

[2] C. Tu, J. Liang and T. D. Tran. "Adaptive Runlength Coding". IEEE Signal Processing Letters, vol. 10, no. 3, pp. 61-64, 2003.

[3] I. Witten, R. Neal and J. Cleary. "Arithmetic Coding for Data Compression". Communications of the ACM, vol. 30, pp. 520-540, 1987.

[4] A. Deever and S. S. Hemami. "What's Your Sign? Efficient Sign Coding for Embedded Wavelet Image Coding". In Proceedings of IEEE Data Compression Conference (DCC), pp. 273-282, 2000.

[5] P. G. Howard and J. S. Vitter. Images and Text Compression, chapter Practical Implementations of Arithmetic Coding. Kluwer Academic Publishers, 1992.
[6] P. G. Howard, L. Bottou and Y. Bengio. "The Z-Coder Adaptive Binary Coder". In Proceedings of IEEE Data Compression Conference (DCC), 1998.

[7] A. Moffat, R. M. Neal and I. H. Witten. "Arithmetic Coding Revisited". ACM Transactions on Information Systems, vol. 16, no. 3, pp. 256-294, 1998.

[8] W. B. Pennebaker, J. L. Mitchell, G. G. Langdon and R. B. Arps. "An Overview of the Basic Principles of the Q-coder Adaptive Binary Arithmetic Coder". IBM Journal of Research and Development, vol. 32, no. 6, pp. 717-726, 1988.

[9] C. Tu and T. D. Tran. "Context Based Entropy Coding of Block Transform Coefficients for Image Compression". IEEE Transactions on Image Processing, vol. 11, no. 11, pp. 1271-1283, 2002.

[10] D. Marpe, H. Schwarz and T. Wiegand. "Context-Based Adaptive Binary Arithmetic Coding in the H.264 / AVC Video Compression Standard (invited paper)". IEEE Transactions on Circuits and Systems for Video Technology, vol. 13, no. 7, pp. 620-636, 2003.

[11] D. Marpe, G. Blättermann, G. Heising and T. Wiegand. "Video Compression Using Context-based Adaptive Arithmetic Coding". In Proceedings of the IEEE International Conference on Image Processing (ICIP), San Fransisco, CA, USA, October 2001.

[12] T. C. Bell, J. G. Cleary and I. H. Witten. Text Compression. Prentice Hall, Englewood Cliffs, NJ, USA, 1990. 